\title{
Öffentliche Meinung im System polyzentrischer Herrschaft
}

\author{
von Udo Di Fabio
}

\begin{abstract}
Die öffentliche Meinung als demokratische Metaebene verfassungsstaatlicher Herrschaftsformen sieht sich mit zwei Strukturproblemen konfrontiert: Einerseits führt die zunehmend dezentrale und vernetzte Form der Massenkommunikation zu einer Abkehr von publizistischen Verantwortungshierarchien, andererseits wird das klassische Verständnis einer demokratischen Öffentlichkeit durch die Internationalisierung des Regierungsstils und das damit einhergehende Fehlen einer klar definierten öffentlichen Bühne politischer Auseinandersetzung in Zweifel gezogen. Dieser Beitrag geht der Frage nach, ob die öffentliche Meinung an diesen Herausforderungen scheitern muss oder ob Anpassungen möglich sind. Dazu werden zunächst moderne Vorstellungen von der demokratischen Öffentlichkeit skizziert, um darauf aufbauend die Auswirkungen erkennbarer Fragmentierungen politischer Herrschaft genauer zu untersuchen. Abschließend wird die Rolle der Medienöffentlichkeit im Netz postmoderner Kommunikation diskutiert und u. a. darauf hingewiesen, dass eine dezentralisierte Massenkommunikation keinesfalls nur in herrschaftsfreien Räumen denkbar ist, mithin die Frage nach der Legitimation und Kontrolle offener oder latenter Herrschaftsausübung zunehmend an Bedeutung gewinnt.
\end{abstract}

Public opinion as a meta-level of democratic forms of constitutional rule is faced with two structural problems: on the one hand, an increasingly decentralised and networked form of mass communication leads to a decline of medial hierarchies of responsibility. On the other hand, the classical interpretation of a democratic public opinion is challenged by a growingly internationalised style of governing which leads to a lack of clearly defined public spaces for political debate. This contribution examines whether public opinion is bound to falter in the face of these challenges or whether adaptation is possible. First, modern perspectives on the form and function of public opinion are traced in order to, second, discuss the effects of an increasing fragmentation of political rule. This leads, third, to a concluding examination of the role of medial public opinion in networked communication and, among others, the insight that decentralised mass communication need not necessarily occur in anarchic spaces and that, therefore, the question of who legitimises and controls those who openly or secretly exert (political) influence in these spaces will come to assume growing importance. 


\section{I. Öffentliche Meinung: eine staatsrechtliche Fiktion?}

Die öffentliche Meinung als Institution moderner Verfassungsstaatlichkeit ist neben Wahlen und Abstimmungen die anerkannte Basis der Legitimation politischer Herrschaft. ${ }^{1}$ Sie ist maßgebliche Voraussetzung für die praktische Möglichkeit von Demokratie. Doch in den Lehrbüchern des Verfassungsrechts wird hier, wenn nicht eine Idylle zugrunde gelegt, so doch mit einer normativen Konstruktion gearbeitet, die sich für die Beobachtung des Tatsächlichen eigentümlich immunisiert. ${ }^{2}$ Seit langem lebt das Recht mit der prominenten Kritik der Sozialphilosophie, die im Recht gepflegte Vorstellung von öffentlicher Meinung sei lediglich eine „staatsrechtliche Fiktion“. ${ }^{3}$ Diese Diagnose mag vor einem halben Jahrhundert noch zu früh gekommen sein, heute aber bricht die Realität postnationaler und womöglich auch postmoderner Wirklichkeiten über eine Staatsrechtslehre herein, die in zentralen Fragen ihres Fachs verstummt oder desorientiert ist. Zwei gravierende Entwicklungen machen die Frage nach dem Schicksal der öffentlichen Meinung dringlich. Die technische Entwicklung vom Druckwerk zum Bildschirm, von der Ordnung der publizistischen Verantwortungshierarchie hin zu einer dezentralisierten Netzwerkkommunikation mit mehr spontaner denn strategischer Ordnungsbildung, erweitert den Informationshorizont, verdichtet Kommunikation, nimmt ihr aber auch die Möglichkeit zur Mitte, beschleunigt und entzeitlicht zugleich, wertet professionalisierte Zugänge ab, macht verlegerischen und journalistischen Berufsethos in der Tendenz entbehrlich. Die dezentralisierte global angelegte Netzöffentlichkeit entfaltet zwar wenig strukturelle Sicherheiten für Validität, kompensiert dies aber ein ganzes Stück weit durch Offenheit, Zugänglichkeit und Vielheit der Interaktionen, die zwar häufig ohne individualisierbare Verantwortungszurechnungen sind, aber in der Summe ein beachtliches kritisches Kontrollpotential ergeben. Was das für die juristische

1 Habermas, J.: Strukturwandel der Öffentlichkeit: Untersuchungen zu einer Kategorie der bürgerlichen Gesellschaft, Frankfurt/M., 1990, 344.

$2 \mathrm{Zu}$ diesem Prozess der Immunisierung zählen nicht nur diejenigen, die Kategorien wie die Meinungsund Pressefreiheit weiter so formulieren, also wären wir, was westliche Demokratien angeht, im 19. Jahrhundert und müssten mit Zensurbehörden rechnen, während schon längst postmoderne Mechanismen der Selbstlimitierung und einer selbstbezüglichen Operationslogik im Meinungssystem das Ruder übernommen haben. Es gehören auch diejenigen zum Vorgang der Immunisierung, die ihre Perspektive vom verfassungsrechtlich Normativen zu einer mehr deskriptiven Perspektive und politischen Utilitaritätserwägungen hin verschieben und dann von verfassungsrechtlichen Prinzipien nur noch geschmeidige Anpassungen an eine ,,neue Wirklichkeit“ verlangen, die von einem wissenschaftlich, politisch und wirtschaftlich geknüpften Netz konstruktiv gezeichnet und bestätigt wird.

3 Habermas, J., a. a. O., 347. 
Annahme einer den politischen Prozess beobachtenden öffentlichen Meinung bedeutet, ist noch ungeklärt.

Gleiches gilt für die zweite große Veränderung unmittelbar im politischen System, die zwar schon lange wahrgenommen wird, aber noch immer unbewältigt bleibt, und zwar im Blick auf die Wahrung und Fortentwicklung des Demokratieprinzips. Die dauerhafte Verbindung von Staaten in internationalen Organisationen und ein diplomatisch-gubernativ geprägtes Regierungssystem des ausgehandelten Konsenses ändert die Herrschaftsbasis für das demokratische Modell. Schon in den fünfziger Jahren, noch bevor die europäische Integration richtig Fahrt aufnahm, noch bevor der UN-Sicherheitsrat seine heute vertraute Stellung erwarb, noch bevor die Welthandelsrunden (GATT/WTO) verstetigt wurden, diagnostizierte die bundesrepublikanische Politikwissenschaft mit Wilhelm Hennis an der Spitze die Erosion der Opposition, das „Versickern“ der ernsthaften Opposition, die Herausbildung eines konsensual weitgehend befriedeten Blocksystems, ${ }^{4}$ Nivellierung und Egalisierung. Dafür gab es innere Gründe der sozialen Marktwirtschaft, der vernünftigen Anpassung der Politik an den Entwicklungspfad der sich wieder globalisierenden Wirtschaft, gerade auch im Blick auf einen liberalen, verhandelten Korporatismus (Sozialpartnerschaft) aber immer mehr auch die Tendenz zum Regieren jenseits des Nationalstaates. ${ }^{5}$

Die Verdichtung teils notwendiger, teils gewollter Kooperationsformen überstaatlicher, zwischenstaatlicher und auch innerstaatlicher Art lasten auf dem Demokratiemodell, das dafür nicht gemacht scheint; die kooperative Verdichtung bis zu einer exekutiven Netzwerkstruktur ist relativ gering in Zentralstaaten, größer in Bundesstaaten und sehr groß im europäischen Staatenverbund. ${ }^{6}$ Das Zusammenspiel der Träger öffentlicher Gewalt untereinander und mit darauf ausgerichteter Verbände (Wirtschafts-, Sozialverbände oder NGOs) wird zur Notwendigkeit eines ausgesprochen steuerungswilligen Politikverständnisses, aber auch zum Problem demokratischer Legitimation und öffentlicher Repräsen-

4 Aufgegriffen vom zurückgezogenen Sensor kommender Dinge Carl Schmitt, der Otto Kirchheim und vor allem Hennis, W.: Parlamentarische Opposition und Industriegesellschaft, in: Gesellschaft - Staat Erziehung, 1/5 (1956), 205-222. zitiert, siehe den Nachtrag von 1958 zur Abhandlung ,Weiterentwicklung des totalen Staates in Deutschland“, in: Schmitt, C.: Verfassungsrechtliche Aufsätze aus den Jahren 1924-1954, 4. Aufl., Berlin, 2003, 366. Siehe auch Hennis, W.: Ende der Politik - Krisis der Politik in der Neuzeit, in: Merkur, 25 (1971), $509 \mathrm{ff}$.

5 Zürn, M.: Regieren jenseits des Nationalstaates, Frankfurt/M., 1998, 294 ff., der eine Tendenz zur thematischen Fragmentierung der Politik hervorhebt.

6 Di Fabio, U.: Das Recht offener Staaten, Tübingen, 1998, 143. 
tation. $^{7}$ Für die segmentierten, national aufgestellten öffentlichen Meinungen geriet die Internationalisierung des Regierungsstils zu einem Dilemma, weil keine Bühne der Mitte nach einem einfachen Schematismus (Regierung/Opposition) beobachtet werden konnte, sondern nur die Erfolgsmeldungen oder deren Ausbleiben in der Folge von Regierungskonferenzen. Anstelle des binären Schematismus von Regierung und Opposition in nationalen Parlamenten trat verstärkt eine ganz neue binäre Schematisierung nach dem Muster „Verhandlungsergebnis oder kein (resp. unzureichendes) Verhandlungsergebnis“. Damit wird die öffentliche Meinung auf das Fortschrittsparadigma von Verhandlungssystemen umgestellt und sachlich präformiert. Presse und Rundfunk können sachlich nur noch beschränkt räsonieren, in begrenzten Teilthemen zwar nach einem symbolischen Tribut verlangen, aber kaum noch eine systematische Opposition formulieren oder publizistisch eine bestehende politische Opposition unterstützen. Im Ergebnis schauen die Bürger auf einen Fernsehmoderator, der ihnen erklärt, welche Verhandlungsfortschritte ein Klima- oder Finanzgipfel erbracht hat, und wo die Bremser saßen, ob sie überwunden wurden oder ob es ihnen wieder mit nationalen Egoismen gelungen ist, Vernunft und Fortschritt zu behindern. Die kritische Durchdringung dessen, was dort geschieht, sieht anders aus, bricht sich aber auch bei scharfem Blick und guten Willen an der Komplexität konsensualer Kompromisse und verhallt auf den gut gedämmten Fluren der Diplomatie, die jeden Konflikt freundlich ummantelt und nach Verstetigung eines jeden vorzeigbaren Verhandlungsergebnisses sucht - in neuen Verträgen, Absprachen und internationalen Einrichtungen. So wird unaufhörlich am Netz des neuen überstaatlichen Regierungssystems geknüpft und die Öffentlichkeit von entschiedener Mitsprache und auch von der Möglichkeit strukturellen NeinSagens entwöhnt.

Sieht man beide Entwicklungen zusammen, ergibt sich die Frage, ob die öffentliche Meinung als demokratische Metaebene verfassungsstaatlicher Herrschaftsform an den Strukturproblemen scheitert oder ob Anpassung möglich ist. Vielleicht weisen sogar beide Entwicklungen in sich Potentiale auf, die gegen den jeweils anderen Trend auch Rettendes für das Prinzip der öffentlichen Meinung wachsen lassen. 


\section{Die moderne Idee öffentlicher Meinung}

Eine frei gebildete Öffentlichkeit, eine freie öffentliche Meinung sind zentrale Voraussetzungen für Demokratie. Dort, wo Zeitungen bedrängt, zensiert, verboten sind, dort wo Journalisten eingeschüchtert, vorgeladen oder durch nie aufgeklärte Straftaten ums Leben gebracht werden, wo abweichende Meinungsäußerungen ins Gefängnis führen, dort kann Demokratie nicht gedeihen. Warum eigentlich ist das so? Das antike Griechenland hat doch schließlich die Demokratie erfunden, ohne dass in Athen Zeitungsverkäufer standen. Wer in Volksversammlungen von 6000 Menschen direkt das Schicksal der Polis bestimmt, braucht keine gedruckte öffentliche Meinung.

Die Neuzeit verfährt differenzierter, schon weil im Mutterland der liberalen Demokratie, in England, keine Volksversammlungen als Ekklesia möglich waren. Hier ging es darum, eine bürgerliche Gegenmacht zu der mit dem Absolutismus liebäugelnden Krone zu etablieren und dem Parlament und den in ihm ringenden Kräften eine Stimme zu verleihen. Die moderne Presse ist insofern ein Ergebnis der Glorreichen Revolution des 17. Jahrhunderts und der mit ihr de facto eingeführten parlamentarisch verantwortlichen Regierung.

Im Übergang zum Jahrhundert der Aufklärung entsteht dann die Vorstellung, dass ein bürgerliches Publikum, das in Handel, Handwerk, Landwirtschaft zu Besitz gelangt ist und Träger der Bildung wird, seine Meinung als Ergebnis freier Diskussion und vernünftigen Argumentierens als politisch bedeutsamer Faktor zum Ausdruck bringt: in Zeitungen, die kühle Nachrichten und kritische Sachkommentare verbinden, die aber auch mit Polemik und Demagogie zu gefürchteten Mitspielern des politischen Prozesses werden. Die public opinion wird zu einer festen Größe im politischen Entscheidungsprozess, sie wird im Grunde Teil des politischen Herrschaftssystems, aber in besonderer Verbindung zu wirtschaftlichem Renditeinteresse, wissenschaftlichem Wahrheitsstreben, ethischer Orientierung und nicht zuletzt lebenspraktischen Einstellungen der Leser.

Die ernsthafte Presse sieht sich als unabhängiger Teil des politischen Prozesses, so wie Richter sich als unabhängige dritte Gewalt im Staat verstehen. Beide Gewalten handeln und urteilen im Namen des Volkes, beide kontrollieren die zur Gestaltung berufenen politischen Kräfte der Legislative und der Exekutive. In dieser Zeit knapp vor der Französischen Revolution entsteht der Beruf des Journalisten, der sich, fachlich versiert und vom Ethos des Aufklärers beseelt, als Auge, Ohr und Mund einer Öffentlichkeit versteht, die aus der privaten Lebenswelt heraus ihre Vernunftmaßstäbe findet. Die Symbiose eines Verlegertypus, 
der Geschäftsinteresse und öffentliche Wirkung in den Mittelpunkt rückt, und der Journalisten, denen es weniger um Rendite, sondern um die öffentliche Rolle der Presse geht, um Beobachten, Berichten und Aufklären: Beides verschmilzt zu dem, was man zuerst in England ,fourth Estate“, vierte Gewalt, nannte.

Unsere Vorstellung geht heute auch dahin, dass alles politisch Bedeutsame in Nachrichten, Tageszeitungen und politischen Magazinen transparent wird. ${ }^{8}$ Die Presse ist die Metaebene des politischen Prozesses, eine Reflexionsinstanz, in der Politik für den Bürger - und auch für viele Politiker - überhaupt erst ihre Einheit, ihre wahrnehmbare Gestalt findet. Das politische Geschehen, also die Gesetzgebung, die Ämtervergabe, Verwaltungsentscheidungen, wären für den Bürger in ihrer Komplexität ohne Presse und Rundfunk, ohne journalistische Aufbereitung und Systematisierung schlechterdings nicht zu verstehen, nicht als irgendwie geordnete Einheit erlebbar. Diese Einheit aber braucht eine Mitte, einen Ort und eine institutionelle Entsprechung wie es das Parlament als Repräsentationsorgan des Volkes ist. Diese Einheit wird aber ihrerseits zu einer rechtlich zwar noch verbindlichen und auch dem praktischen Erwartungsverhalten der Bürger noch entsprechenden, aber ansonsten mehr und mehr fiktiven Vorstellung. Die Europäische Union und die internationale Politik lassen sich schwerlich als segmentiertes System im klassischen Sinne völkerrechtlich verkehrender Staaten verstehen. Es entsteht vielmehr ein Netzwerk polyzentrischer Handlungseinheiten. Die Staaten sind wie die internationalen Organisationen und europäischen Organe zwar bedeutsame und Halt gebende Knotenpunkte im Netzwerk, aber ihre Handlungsmöglichkeiten und Beziehungen können nicht als Summe einer komplizierten Bi- oder Multilateralität verstanden und öffentlich abgebildet werden. Das Netzwerk hat seine eigene Handlungslogik und findet seine Einheit nicht so sehr an einem Ort (New York für den UN-Sicherheitsrat oder Brüssel für die EU-Kommission), sondern in der Polyzentrik politischer Organisationen, die sich häufig wie wissenschaftlich angeleitete Verwaltung von Land und Leuten (Helvetius) versteht. ${ }^{9}$ Kann sich öffentliche Meinung darauf einrichten oder entkoppelt sie sich als Vierte Gewalt vom politischen System und wird in der Tendenz Feuilleton?

8 Luhmann, N.: Die Realität der Massenmedien, 3. Aufl., Wiesbaden, 2004, 14, hält das in kantischer Terminologie für eine transzendentale Illusion.

9 Fach, W.: Das Verschwinden der Politik, Frankfurt/M., 2008, 175. 


\section{Einheitsverluste und Fragmentierungen politischer Herrschaft}

\section{1. „Gesellschaft der Individuen“ und „Gesellschaft der Netzwerke“: Antipoden oder Komplementäre?}

Im internationalen Politiksystem kann man zwei komplementäre Trends ausmachen. Zum einen zeigt die politische Öffentlichkeit in nationalen oder manchmal sogar in regionalen Kulturräumen eine erstaunliche Beharrungskraft. Die Bühnen des politischen Geschehens bleiben national in ihrer Sprache, mit ihrer Syntax und ihren Konnotationen, auch mit ihrer politisch-kulturellen Semantik und historischen Symbolik. Das vergrößert allerdings die Kluft zum überstaatlichen oder nach innen föderalisierten Entscheidungssystem, weswegen die politische Öffentlichkeit häufig nur noch Zaungast des Geschehens zu sein scheint, allerdings mit einem gewissen Druckpotential auf die eigene Regierung. Wegen des überstaatlicher werdenden Regierungsstils rücken die nationalen Öffentlichkeiten zwar näher aneinander, beobachten sich auch stärker, bleiben aber in der Anordnung zueinander segmentiert. Die wachsende Weltgesellschaft bleibt insofern im Staatenverkehr und unter Geltung des Prinzips souveräner Gleichheit durchaus segmentiert, in gleichen Handlungseinheiten nebeneinander gestellt. Darüber wölben sich allerdings verstärkt die überstaatlichen Formen des Regierens, es werden autonome Machtquellen gebildet, die Staaten installieren dauerhafte oder spontane Systeme des Interessenausgleichs und vertiefen die Kooperation mit verfestigten und ausgebauten internationalen Organisationen. Entstanden ist neben den fortbestehenden üblichen internationalen Machtlagen und den üblichen bürokratischen Verselbständigungstendenzen ${ }^{10}$ inzwischen ein verfestigtes polyzentrisches Verbundsystem, ein Netzwerk, das von kontrolliert verliehener Autonomie zu immer mehr Unabhängigkeit strebt, zu einem Selbststand aus eigener, und nicht verliehener Legitimation. Es entspricht gerade der auf kalkulierte Unklarheit angelegten Postmodernität, dass den insoweit besonders weit gelangten Unionsorganen dabei der letzte Schritt - etwa die Abkehr von der völkervertragsrechtlichen Grundlegung und der distinktiven Gründung eines europäischen Bundesstaates - bislang aber vorenthalten bleibt. Daraus folgt ein instabiles Gleichgewicht; instabil, weil es statische Elemente verbindet mit dynamischen, so dass eine Gleichzeitigkeit zu beobachten ist von dynamischer Autonomisierung der Netzwerke und pragmatischer Schwebelage der letzten

10 Allgemein: Mayntz, R./Nedelmann, B.: Eigendynamische soziale Prozesse, in: Kölner Zeitschrift für Soziologie und Sozialpsychologie, 39 (1987), $633 \mathrm{ff}$. 
Konstruktions- und Machtfragen: Wer hat das letzte Wort? Was ist primär und was ist sekundär? Die Moderne konstruiert nach Plan und deduziert aus Axiomen, die die individuelle und gemeinsam betätigte Vernunft in der Mitte hält, die Postmoderne ist zieloffen konzipiert, lernt an sich selbst, zielt auf fraktionierte Ergebnisse und Fortschritt, kommt ohne Mitte aus, spielt mit den weiter existierenden und den neuen Zentren um ein neues Gleichgewicht der Macht.

Die Abkehr von klaren Grenzen und Unterscheidungen scheint sachnotwendig, wenn man eine kontinentale und globale Ordnung stiften und über das Territorium des Staates hinausgreifend politisch gestalten will. Aber so einleuchtend, ja so unabweisbar ein solches Streben ist, so deutlich stehen dann doch auch die neuzeitlichen Staats- und Modernitätsmuster als Hindernis im Weg: mit ihrer Einheitsidee von Vernunft, die auf einzelne Subjekte rückführbar sein muss und der periodischen Möglichkeit demokratischer Richtungsentscheidung. Keiner der nach sachlichen internationalen und europäischen Lösungen sucht, will Demokratie oder Kontrolle durch die öffentliche Meinung abschaffen, im Gegenteil wird angestrengt nach Funktionsäquivalenten gesucht. In einem sektoral gegliederten politischen Klima verselbständigen sich Sachprobleme, wenn organisierte Interessen mit politisch-moralischen Präferenzregeln und der Steigerungslogik von Erfolgsnachweisen gekoppelt werden. Sachgebiete und Themenkomplexe, die von kognitiven, volitiven und moralischen Präferenzen vorstrukturiert sind (Klima, Umwelt, Wachstum, Europa, IT, Gleichstellung, Integration, Gesundheit, Mobilität) werden personell und institutionell betreut, wissenschaftlich beraten, von Interessengruppen mitgestaltet und beobachtet, deren Informationspolitik dann als präformiertes Wissen in den Prozess der öffentlichen Meinung einfließen lässt. Die ebenfalls - und zwar spätestens seit der Aufklärung - als moralisch überlegen fixierte Fortschrittsdynamik innerhalb der durch die Sachfrage begrenzten Fraktion lässt dann (auch aus der Perspektive der jeweiligen medialen Teilöffentlichkeit) institutionelle Fragen und konzeptionelle Einheitsentscheidungen als sperrige, ja sogar als irrationale Einwände erscheinen.

Der Übergang zur postmodernen Staatlichkeit wird von Ladeur als Übergang von klaren Unterscheidungen und Institutionen hin zu einer Gesellschaft der Netzwerke gesehen, die Verschleifungen an die Stelle klarer Grenzziehungen setzt. ${ }^{11}$ Damit unlöslich verbunden sind sachbereichsspezifische Fragmentierungen, die im politischen System einheitliches Handeln erschweren, weil sie selbstbezügliche Steigerungsprozesse freisetzen, bei denen jedes von der öffent- 
lichen Meinung aufgegriffene Problem willkommener Anlass für die Präsentation neuer Programme wird, weil in einer nur schlecht kaschierten illiberalen Tendenz alles in der politischen Perspektive (,, Seeing like a State ") wahrgenommen wird. ${ }^{12} \mathrm{Im}$ gesellschaftlichen Großbereichen wie Sozialverwaltung, Medizinversorgung, Energiepolitik, Bildung, Wissenschaft, Tarifvertragspolitik, Welthandelsforen, Klimaschutz, Arbeitsmarktregulierung entstehen Netzwerke mit organisierten Teilöffentlichkeiten, die Wissen und kritische Potentiale scheinbar selbstbezüglich steuern, bei näherem Hinsehen, aber eine Verschleifung von Wissen und Interessen darstellen, die mit politischer Steuerungsabsicht nach außen tritt und von einer immer noch am Ideal und Bild der Einheit festhaltenden medialen Öffentlichkeit nur eingeschränkt erreichbar ist. Jedes Fragment besitzt über seine inhärente Funktionalität hinaus adaptive Mechanismen, die Verbindung halten mit moralischen Präferenzen und Bedürfnisses (Interessen) des Publikums. Das Gesundheitssystem etwa gehorcht einem moralisch verankerten Gleichversorgungsgebot und lässt alle hoffen, auf höchstem Niveau vor Krankheit und Tod geschützt zu werden. Klimapolitik wahrt die Harmonie der Schöpfungsordnung, sichert bezahlbare Energie, schützt vor Naturkatastrophen. Das Bildungssystem ist gut, weil Wissen besser als Nichtwissen ist, es macht junge Menschen fit für die Erfordernisse der (post)modernen Welt, damit ihre Produktivität allen nutzt und nicht ihre aus Ignoranz folgende Devianz allen schadet.

Die strukturelle Intransparenz und Selbstabgrenzung der fragmentierten Netzwerke und ihre zunehmende Hybridisierung hoheitlichen und privaten Handelns ${ }^{13}$ wird zum Kennzeichen, aber auch sogleich zum Problem postmoderner Gesellschaftsorganisation. Die Fragmentierung entsteht weder zufällig noch ohne Gewinn, auch für den einzelnen Bürger in seinem Freiheitsanspruch. Weil das Gesundheitssystem komplex organisiert ist, muss es nicht schlecht sein. Die Europäische Union macht öffentliche Zurechnung und Einheitsrepräsentationen gewiss schwierig, aber ihr Gewinn auch für die (koordinierte) Durchsetzung demokratischer Willensbildung aus den staatlichen Primärräumen ist im Gewicht weit größer. Es kommt also darauf an, wie die aus der freien und gleichen Person deduzierte Ordnung mit Netzwerken operiert, wie klare Grenzen formuliert und Einflusspotentiale verteilt sind oder kurz: wie die Gesellschaft der Individuen mit der Gesellschaft der Netzwerke gekoppelt wird.

12 Ebd., 179.

13 Ebd., 185. 


\section{Postmodernität ernst genommen}

Doch bevor nach Kopplungsstrategien auch für den kognitiven, moralischen und demokratischen Anspruch der öffentlichen Meinung gegen die postmoderne Fragmentierung politischer Organisationswelten gesucht wird, muss zunächst gefragt werden, ob eine solche Kopplung noch gelingen kann, wenn das Rationalitätsparadigma bereits postmodern verschoben sein sollte. Der Begriff ,postmodern" hat in diesem Zusammenhang nicht nur als Ornament, sondern im vollen begrifflichen Ernst seine Berechtigung, wenn das human-rationalistische Paradigma der Neuzeit konzeptionell überschritten wird. Die Neuzeit hatte den einzelnen Menschen nicht nur als Gattungstypus, sondern sowohl normativ wie faktisch in den Mittelpunkt jeder Ordnung gerückt. Das mirandolische Freiheitsaxiom $^{14}$ jener weltlichen Inbesitznahme der biblischen Botschaft von der Gottesebenbildlichkeit hatte jedem einzelnen Menschen einen angeborenen Anspruch auf frei gewählten und selbst verantworteten Lebensentwurf gegeben. Art. 1 Abs. 1 GG und Art. 2 Abs. 1 GG und Art. 3 Abs. 1 GG machen das unübertrefflich deutlich: Würde des Menschen und freie Entfaltung seiner jeweiligen Persönlichkeit im Rahmen der für alle geltenden allgemeinen Gesetze, die von allen in gleicher Weise frei gegeben werden.

Englische Verfassungskämpfe des 17. Jahrhunderts, amerikanische Unabhängigkeitserklärung und französische Menschenrechtserklärung wie auch die deutsche Paulskirchenverfassung und dann im 20. Jahrhundert die UN-Menschenrechtserklärung geben Zeugnis von der Wirkkraft dieses natur- und vernunftrechtlich konkretisierten Paradigmas. In dieser Tradition stehen die westlichen Verfassungstexte, diese Tradition hat das Lissabon-Urteil des Bundesverfassungsgerichts vom 30. Juni 2009 bekräftigt, ${ }^{15}$ weil diese Matrix dem Verfassungstext klar zugrunde liegt. Aber es kann nicht aussichtsreich sein, scheinbar unbeeindruckt modern zu bleiben, wenn alle Weichen auf einen Epochenwechsel gestellt sind, zumal niemand weiß, wie die Signatur der neuen Epoche ausfällt. Wird die tradierte Realität der öffentlichen Meinung und die mit ihr gekoppelte verfassungsstaatliche Formtypik der neuen Postmodernität schlicht zu weichen haben? Ist Modernität heute konservativ oder gar schon rückwärtsgewandt? Kann es nur darum gehen, die hybriden Netzwerke von privaten und öffentlichen Akteuren unter der Gesetzesbindung und in demokratischer Verantwortlichkeit zu halten

14 Di Fabio, U.: Gewissen, Glaube, Religion, 2. Aufl., Berlin, 2009, 52 ff.

15 BVerfG, Urt. v. 30.06. 2009, 2 BvE 2/08 u.a. (Vertrag von Lissabon), abgedruckt u.a. in: Neue juristische Wochenschrift, 62/31 (2009), 2267-2295, hier Rn. $208 \mathrm{ff}$. 
oder überstaatliche wie föderale Beziehungsgeflechte für demokratische Impulse aus politisch-kulturellen Primärräumen - heute trotz aller transformativen Bindung durchweg noch die Nationalstaaten - offen zu halten?

Was genau markiert den Unterschied zwischen neuzeitlich und modern auf der einen Seite und Postmodernität auf der anderen Seite? Als Faustformel könnte gelten: Modern ist, wer an der subjektiven Vernunft und Freiheit des Einzelnen festhält und postmodern ist derjenige, der in Netzwerken mehr „Vernunft“ am Werk sieht, seien es neuronale, kommunikative, sozialsystemische, wissenschaftliche, rechtliche oder politische. Mit der Neuzeit, also mit Renaissancehumanismus, Rationalismus, Reformation und Aufklärung wird der Mensch zum Individuum, zum Künstler, zur Person, zum Subjekt. Als natürliches Rechtssubjekt und als Quelle für alle Erkenntnisgewissheit und Gewissensüberzeugung wird er mit der Institution der Privatautonomie zur entscheidenden Quelle der Privatrechtsordnung, ${ }^{16}$ mit Descartes zum Erkenntnissubjekt, mit Kant zum Subjekt der praktischen Vernunft und später dann folgerichtig mit Wahlen und Abstimmungen zur Quelle aller demokratischen Legitimation und eines ihm dienenden Rechtsstaates.

Ein halbes Jahrtausend Neuzeit sind der Versuch, eine „Gesellschaft der Individuen ${ }^{\text {“17 }}$ zu bauen, die sich im öffentlichen Raum begegnen und die von eigenen Interessen geleitet, aber mehr noch von ihrer eigenen Einsichtsfähigkeit bestimmt und letztlich nur ihrem Gewissen unterworfen handeln oder von dort aus deliberativ verhandeln. Von der ersten Stunde der Neuzeit an ist dieses humanistische und liberale Ideal gesellschaftlicher Organisation allerdings vehement bekämpft worden, weil der Mensch ,in Wirklichkeit“ schlecht sei, Triebe und Sünde viel stärker als die Wirkkraft der sittlichen Vernunft wären, also deshalb das Gemeinwohl, der Frieden und der Wohlstand doch wohl anders zur Geltung gebracht werden müssten, anders als dies durch die Summe der Egoismen, Vorurteile und Irrtümer der vielen Einzelnen möglich wäre. Die Fürsten, die Geistlichen, die Gelehrten warnten vor Anarchie und Zügellosigkeit, vor Zerstörung einer größeren Vernunft, die nicht dem Einzelnen zugänglich sei, sie wollten

16 Wobei deren Bedeutung im Verlauf der Entwicklung meist erheblich unterschätzt oder verkannt wurde, siehe dazu etwa Mestmäcker, E.-J.: Franz Böhm und die Lehre von der Privatrechtsgesellschaft, in: Riesenhuber, K. (Hg.): Privatrechtsgesellschaft, Tübingen, 2007, 35 ff., hier 38.

17 Ebd., 191. 
eine Würde und Harmonie des Ganzen bewahren, die dem Individuum erst seinen Ort und seinen Glanz verlieh. ${ }^{18}$

In der Postmodernität kehrt vieles davon in neuem Gewand zurück. Wird aus dem „,eelestialen Globus“ des 16. Jahrhunderts das Klimagleichgewicht des 21. Jahrhunderts? Aus der alten Antithese zur Moderne wird die neue These der Postmoderne. Schon Verteidiger der neuzeitlichen Vernunft wie John Rawls oder Jürgen Habermas haben als Ort der Vernunft auf pluralisierte Öffentlichkeiten ${ }^{19}$ und die subjektlose Intersubjektivität des Diskurses gesetzt. Das allerdings steht anders als bei Kant gegen die ,republikanische Vorstellung, Selbstgesetzgebung müsse auf konkrete einzelne Individuen heruntergerechnet werden können“ “ ${ }^{20}$ Nicht Menschen, sondern Kommunikationen ${ }^{21}$ - so die Vorstellung - formen das Netz der politischen Öffentlichkeit. ${ }^{22}$ Der jahrzehntelange Gegenspieler einer solchen Theorie kommunikativen Handelns Niklas Luhmann hat in seiner „Gesellschaft der Gesellschaft" scheinbar gleich ganz auf das Subjekt und den Menschen verzichtet, in dem er sich der Autopoiesis von gesellschaftlichen Funktionssystemen widmet. Und nur wer seine Fähigkeit zur Selbstironie gerade in der Wahl des Theoriedesigns richtig einschätzt, wird merken, dass er womöglich viel entschiedener als Habermas die Neuzeit verteidigt, schon indem er deren Herausforderer im Abstraktionsniveau weit überbietet.

18 In Bertolt Brechts „Leben des Galilei“ gibt es dazu im Blick auf die Freiheit der Wissenschaft eine Schlüsselszene: Die Herren der Florentiner Universität steigen über das zerbrochene Ptolemäische Holzmodell hinweg in Galileis Studierzimmer, damit sie endlich durchs Fernrohr auf den Jupiter schauen. Denn wenn sie die Jupitermonde beobachten, werden sie sehen, das die Monde hinter dem Jupiter verschwinden, was die Kreisbahn belegt und unverträglich wäre mit der Vorstellung einer Fixierung der Gestirne an Kristallschalen. Wenn sie schauen, müssen sie sich auf die unerbittliche Logik wissenschaftlicher, auf Beobachtung gestützter Welterklärung einlassen und kapitulieren. Aber sie tun es nicht. Der Philosoph will zuerst einen gelehrten Disput, weil die Wissenschaft Teil einer größeren Ordnung und eben keine selbstbezügliche ist. „Das Weltbild (so sagt er) des göttlichen Aristoteles mit seinen mystisch musizierenden Sphären und kristallenen Gewölben und den Kreisläufen seiner Himmelskörper und dem Schiefenwinkel der Sonnenbahn und den Geheimnissen der Satellitentafeln und dem Sternenreichtum des Katalogs der südlichen Halbkugel und der erleuchteten Konstruktion des celestialen Globus ist ein Gebäude von solcher Ordnung und Schönheit, dass wir wohl zögern sollten, diese Harmonie zu stören.“ Gegen die von den Kirchvätern anerkannte göttliche Autorität des Aristoteles kommt Galilei nur mit dem Argument der „Wahrheit“, also jener ungebundenen selbstbezüglichen Freiheit der Wissenschaft. Darauf reagiert der bislang so autoritativ gelassene Philosoph der florentinischen Universität geradezu emotional und sagt „,wild“: „Herr Galilei, die Wahrheit mag uns zu allem möglichen führen!“‘.

19 Rawls, J.: Das Recht der Völker, Berlin, 2002, $167 \mathrm{ff}$.

20 Palazzo, G.: Die Mitte der Demokratie, Baden-Baden, 2002, 96.

21 Es geht um die Vorstellung eines für maßgeblich erklärten kommunikativen Handelns unter dem Präformationsdruck der Sprachgrammatik und der in sie eingelassenen Vernunftpotentiale, Habermas, J.: Theorie des kommunikativen Handelns, Frankfurt/M., 1981.

22 Palazzo, G., a.a. O. 
Vieles an neuen Formen gesellschaftlicher Hybridisierung von öffentlicher Gewalt und privaten Akteuren, von Wissen, Politik und Moral sowie die zunehmende wechselseitige Instrumentalisierung von Funktionssystemen deuten darauf hin, dass sich das fragmentale Organisationsprinzip über das fortbestehende moderne Prinzip der funktionalen Differenzierung schiebt. Politik ist heute ebenso stark abhängig von Wissenszuflüssen aus der Wissenschaft ${ }^{23}$ wie von den Sachnotwendigkeiten des Wirtschaftswachstums, sie ist zudem im Griff der thematisch vernetzten Teilöffentlichkeiten. Die Wissenschaft ihrerseits steht zunehmend im Banne von Ökonomisierung und Politisierung. Die Wirtschaft hat nicht das Erbe der Politik als Zentrum der Gesellschaft angetreten, ihr Operationsprinzip durchwirkt zwar die Gesellschaft immer deutlicher, aber sie selbst steht dem System polyzentrischer Herrschaft manchmal orientierungslos gegenüber: Welchem großen Automobilhersteller wollen wir für die nächsten drei Jahrzehnte eine Prognose ausstellen, wie er mit der Veränderung politischer Rahmenbedingen die Antriebstechnik betreffend, Währungsrisiken und der staatlichen Förderung neuer oder alter Wettbewerber wird umgehen können?

Soziologie und Politikwissenschaft werden alle Hände voll zu tun haben mit der Frage, ob das postmoderne System polyzentrischer Netzwerkherrschaft die funktionale Ausdifferenzierung der Gesellschaft allmählich durch fragmentierte Entdifferenzierungen ersetzt oder doch umformt, wodurch aber der Weg zu einer wahrheitsorientierten und kritischen Beobachtung der Gesellschaft im Medium öffentlicher Meinung noch unwahrscheinlicher wird.

\section{Medienöffentlichkeit im Netz der postmodernen Kommunikation}

Die Veränderungen im global vernetzten Kommunikationsverhalten könnten eine nachdrückliche Bestätigung der Tendenz zur Fragmentierung und der Übertragung von - ursprünglich beim Individuum ressortierender - Vernunft auf emergente Netzintelligenz sein. ${ }^{24}$ Die Individualisierung der Informationsströme scheint in der Tat die übliche durch Presse und Rundfunk repräsentierte Mitte

23 Der europäische Klimaschutz folgt weitgehend den Einschätzungen des wissenschaftlich besetzten Weltklimarates und den entsprechenden Adaptionsmodulen für policymakers, siehe etwa IPCC: Climate Change 2007: The Physical Science Basis - Contribution of Working Group I to the Fourth Assessment Report of the IPCC (Summary for Policymakers), 2007.

24 Siehe insofern bereits Mayntz, R.: Individuelles Handeln und gesellschaftliche Ereignisse: Zur MikroMakro-Problematik in den Sozialwissenschaften, in: Wie entstehen neue Qualitäten in komplexen Systemen? 50 Jahre Max-Plank-Gesellschaft 1948-1998, Göttingen, 2000, 5 ff. 
weiter auszuhöhlen. ${ }^{25}$ „Nachrichten werden so durch Computer von ihrem tatsächlichen Informationszusammenhang getrennt, in einzelne Bestandteile zerlegt, kommerzialisiert, weiterverarbeitet und jedem Nutzer individuell zugestellt - ein journalistischer Albtraum, da keine tiefer gehenden Analysen und Interpretationen von Nachrichten mehr vorgenommen werden, sondern jeder Nutzer mit einer Vielzahl zusammenhangloser, oberflächlicher und belangloser Informationen bombardiert wird“. ${ }^{26}$ Allerdings könnten in Internetforen auch neue Mittelpunktbildungen entstehen, die dann auch zunehmend von Printmedien und Rundfunkprogrammen aufgegriffen oder gefördert werden. Im Netz könnte insofern auch Rettendes wachsen, wenn es hier gelänge, die Abgeschlossenheit von fragmentierten und nach Interessen formierten Teilöffentlichkeiten aufzubrechen.

Das Internet ist ein offenes Meer, in dem spontane Ordnungen entstehen, mächtige Akteure wie „Google“ das Sagen haben, die aber auch nur solange mächtig bleiben, wie sie Spürnasen für Nutzerbedürfnisse und praktisches Talent zu deren Kommerzialisierung entwickeln. Keiner weiß heute, ob die Zeitungen und der Journalismus mit ihren modernen kritischen Vernunftansprüchen sich in dieser digitalen Welt auf Dauer behaupten werden, gleich ob im Wettbewerb oder mit Hilfe von gebührenfinanzierten Rundfunkbetreibern. Unklar ist auch, ob dies in einem Umfang gelingt, die der vertrauten Rolle der Pressefreiheit und der öffentlichen Meinung in demokratischen Systemen entspricht oder ob sich hier nur Residualbereiche für Unterhaltung und Infotainment, für das personelle Casting des politischen Nachwuchses halten.

Viele Internetnutzer halten allerdings solche Fragen schon für schief gestellt. Für sie geht es gar nicht darum, wie die traditionelle Rolle der öffentlichen Meinung auch im Internet gewahrt werden kann. Für sie hat die „digitale Revolution“227 die technischen Voraussetzungen für ein ganz neues Modell der Demokratie eröffnet. Dem klassischen Demokratiemodell, dem alle westlichen Verfassungen verpflichtet sind, lag das bürgerliche Privatrechtsmodell einer Verkehrsgesell-

25 Gemeint ist etwa ein Verschwinden des üblichen Nachrichtenmarktes durch das, was Internetentwickler lakonisch als „Evolving Personalised Information Construct" prognostizieren, siehe Weichert, S./Kramp, L.: Das Verschwinden der Zeitung?, Berlin, 2009, 11.

26 Klinghammer, $W .:$ Epic 2015. Eine düstere Zukunftvision, 19.05. 2008, siehe auch: http://internationaleanimationsfilme.suite101.de/article.cfm/epic_2015\#ixzz0OgCD2scW und http://internationale-anima tionsfilme.suite101.de/article.cfm/epic_2015\#ixzz0OgC7m3bj.

27 Die digitale Revolution wird von vielen keineswegs als Aufbruch, sondern als Abbruch (nicht nur als Traditionsabbruch) verstanden, wobei eine ausgewogene Position schwer zu sein scheint, vgl. zu einem solchen Versuch: Heuser, U.J.: Tausend Welten. Die Auflösung der Gesellschaft im digitalen Zeitalter, Berlin, 2000. 
schaft zugrunde, die auf wirtschaftliche Autonomie mittels Privateigentum, auf private Selbstbestimmung und Persönlichkeitsentfaltung durch Bildung setzt. Das Publikum waren im Ideal die gebildeten Citoyens, deren Speerspitze kritischer Aufgeklärtheit gerade auch die Journalisten sein sollten, deren Unabhängigkeit durch das wirtschaftliche Geschick der Verleger nicht gefährdet, sondern gesichert wird. Ihre Wirkung entfalten sie im Sprachraum einer Nationalgesellschaft, ihre Bühne folgt dem politischen Entscheidungssystem des Staates, der im Verfassungs- und Rechtsstaat dem bürgerlichen Vertragsmodell der Privatautonomie am besten entspricht.

Doch das bürgerliche Privatrechtsmodell war immer dem „linken“ Einwand ausgesetzt, es sei nur formal gleich, in Wirklichkeit privilegiere es die Eigentümer gegenüber den Habenichtsen: Wer genießt denn - wird gefragt - Pressefreiheit, der kleine Club der Journalisten und diejenigen, die sich eine Zeitung oder eine Meinung kaufen können, durch Aktienübernahme oder mit großen Werbeetats? Wie viel gleichheitsgerechter und der Wirklichkeit entsprechender scheint dagegen das Internet, wo jeder seinen Text, sein Bild, seine Musik, seinen Film ins Netz stellen und schauen kann, was daraus wird. Und manche denken, dass wir uns über Demokratie hinausbewegen, weil die ganze Idee der Herrschaft von Menschen über Menschen in einer neuen Architektur fragmentierter Sachwelten, die Herrschaft entpersonalisiert, obsolet wird. Wo keine politische Herrschaft im strengen Sinne mehr ist, bedarf aus keiner Vorkehrungen für die Bewahrung von Volksherrschaft. ${ }^{28}$

Das liberale Grundvertrauen im Blick auf die Entstehung spontaner Ordnungen in neuen Kommunikationsforen könnte erst dann ins Wanken geraten, wenn kommerzielle oder anonym auftretende politische Interessengruppen die scheinbare Anarchie des Netzes für ihre Zwecke geschickt nutzen und sich an die im Netz sichtbar werdende subjektive Emotionalität des Nutzers andocken. Auch Politiker und staatliche Stellen suchen die Möglichkeiten. Sowohl die offene Expressivität des Intimbereichs, die den modernen Unterschied von ,privat“ und „öffentlich" schwinden lässt, wie auch die Anonymität des Netzes hat jedenfalls Konsequenzen für moderne Rechtsinstitutionen, die manchmal unerwartet sind: Wenn man zum Beispiel die Verursacher kinderpornografischer Seiten nicht findet, muss man womöglich die Strafe für den bloßen Besitz schärfen, damit der

28 Crouch, C.: Postdemokratie, Bonn, 2008, 31. Dagegen kann man allerdings mit Heidegger antworten: „Erst im Horchen wird das Hören möglich und Hörbares vernehmlich." Heidegger, M.: Gesamtausgabe Band 85, Vom Wesen der Sprache, Nr. 93, Frankfurt/M., 1999, 111. 
lukrative Markt ausgetrocknet wird: Das ist sachlich wahrscheinlich unausweichlich, kann aber die Proportionen schuld- und tatangemessenen Strafens bedenklich verschieben.

In den Potentialen des globalen Netzes könnte auf nicht allzu ferne Sicht der technische Überschlag endgültig weg vom modernen Zeitalter schlummern. War es nicht die große Leistung der frühen Neuzeit, aus der Anonymität der sozialreligiösen, der ständisch-familialen Netzwerke die Person, das Individuum erstehen zu lassen, als Heros, als Subjekt, als Genius, als Rechtsperson? Warum kennen wir Namen wie Dante, Michelangelo, Descartes oder Rubens, und warum kennen wir kaum je die Namen der Architekten gotischer Kathedralen des Mittelalters? Aber wie ist es heute? Wer schreibt für „Wikipedia“, das nicht nur jeder Schüler als digitales Lexikon ohne zu zögern konsultiert? Warum zeigt sich das Gesicht der Kommunikationsteilnehmer nicht offen im Netz? Der freie Mensch der Neuzeit zeigt sein Gesicht, gibt seinen Namen preis, wenn er die Bühne des öffentlichen Raumes betritt, nur bei Zensur oder Pression gilt Anonymität als legitimes Mittel zur Gegenwehr. Aber wenn die im Netz breit genutzte Möglichkeit zur Anonymität genommen wäre, würde sich womöglich das Potential für emergente Intelligenz und spontane Meinungsbildung fern der organisierten Interessen deutlich mindern, es hieße das Netz stärker an die Kette von Urheberrechten, Zitierusancen, Beleidigungstatbeständen und der Prangerwirkung bei inkorrekten Meinungsäußerungen legen. Es ist gar nicht so, dass die verbundenen Staaten und internationale Organisationen gegenüber dem Internet per se machtlos wären. Aber Demokratien fürchten nicht nur den Druck der übrigens typisch fragmentierten - öffentlichen Meinung im Netz, sondern sie wollen auch den Diktaturen und Autokratien kein Beispiel für die staatliche Lenkung der globalen Informationsfreiheit geben.

Ganz nebenbei ändert die Interaktivität und die technische Modalität des Netzes die Kultur der Alltagserfahrung womöglich grundlegend. Wo alles auf Klick verfügbar scheint, entsteht eine Kultur der solipsistischen Verfügbarkeit: Auch das scheint eher dem Zug in die Postmodernität zu entsprechen. Nicht mehr die Bürger, die mit ihrer Arbeit, ihrem gebildeten Verstand das Publikum als eigentliches Subjekt der öffentlichen Meinung bilden, sondern der ununterbrochene Strom eines Konglomerats aus Kommerz und Emotion, aus Information und Unsinn, aus gesteuerter Ordnung und wildem Zufall wird zum Herrschaftssubjekt, tauscht die neuen Ideale der Direktheit, des unmittelbaren Effekts, auch der totalen Gleichheit des Zugangs gegen den Anspruch, die Welt nach Menschenmaß in einem diskursiven Prozess, mit Mehrheit in einem förmlichen Verfahren 
demokratisch zu gestalten. Aber die Moderne muss sich keinesfalls einer solchen negativ gezeichneten Erwartung ergeben.

Es ist schon falsch, an geradlinig verlaufende Entwicklungen zu glauben. Die Weltgesellschaft besitzt eine Struktur dualer Wirkungskräfte und komplementären Gleichzeitigkeiten. ${ }^{29}$ Mit der Entwicklung des Fernsehens wurde das Verschwinden des Buches und der Zeitungen vorausgesagt, eingetreten ist ihre sich ergänzende Ko-Existenz. Durch die Pluralisierung der Lebensstile wurde das rasche Ende von Ehe und Familie erwartet, aber die Zahl der Eheschließungen demographisch bereinigt - bleibt in etwa konstant, andere Lebensgemeinschaften sind ergänzend an die Seite getreten, ohne dass es darüber zum Kulturkampf gekommen ist. Mit der Entwicklung der Europäischen Union ist das Verschwinden des Nationalstaates vorausgesagt worden. Heute erleben wir, dass die Europäische Union an Gewicht ständig zunimmt, aber weder Bund noch Länder haben ihre Bedeutung und politische Macht verloren, nicht alles ist ein Nullsummenspiel. Die Aufgaben und Ziele internationaler Organisationen weiten sich aus, doch die demokratische Inklusions- und Ordnungskraft demokratischer Staaten bleibt grundlegend. Es entstehen eben neue Gleichzeitigkeiten und Wirkkräfte auf verschiedenen Ebenen. Insofern sind Begriffe wie „polyzentrische Herrschaft" oder „Postmoderne“ sowohl deskriptiv durchaus treffend, aber immer auch Verlegenheitsbegriffe für eine noch nicht hinreichend verstandene Formtypik gesellschaftlicher Differenzierung. Bislang ist es nicht hinreichend gelungen, eine begehbare Brücke zwischen dem normativen Anspruch aufgeklärter Vernunft, humanistischer und demokratischer Gesellschaftsfundierung auf der einen Seite und der Eigenlogik von sozialen Funktionssystemen im Übergang zu fragmentierten, verfestigten Mischformen auf der anderen Seite zu schlagen.

29 Interessante Überlegungen hierzu bei Weber, S.: Die Dualisierung des Erkennens. Zu Konstruktivismus, Neurophilosophie und Medientheorie, Wien, 1996. 